\title{
Botulinumtoxin lindert neuropathische Schmerzen
}

Periphere neuropathische Schmerzen lassen sich durch subkutane Injektionen von Botulinumtoxin (Btx) A bessern. Vor allem Patienten mit Allodynie und geringem thermalem Defizit profitieren.

Experimentelle Untersuchungen der vergangenen Jahre haben gezeigt, das Btx A über seine Effekte auf den Muskeltonus hinaus auch analgetisch wirkt. Ein Team um Nadine Attal vom Institut national de la santé et de la recherche médicale (INSERM) in Boulogne-Billancourt, Frankreich, hat in einer kleinen, doppelblinden und placebokontrollierten Studie untersucht, ob Btx A auch Vorteile für die Behandlung von Patienten mit Neuropathieschmerzen mit sich bringt.

\section{Patienten mit starken Schmerzen}

Beteiligt waren 68 Patienten von zwei französischen und einer brasilianischen Schmerzambulanz mit neuropathischen Schmerzen - meist Schmerzen nach einem Trauma oder einem operativen Eingriff. Die Daten von 66 Patienten gelangten in die Endauswertung. 34 von ihnen hatten subkutane Btx-A-Injektionen in die schmerzende Region erhalten. Dazu war eine Technik adaptiert worden, die zur Behandlung von Hyperhidrose eingesetzt wird. Das Zielgebiet wird dafür gerastert: Im Abstand von 1,5-2cm setzen die Ärzte Injektionen und verabreichen pro Einstichstelle 5 Einheiten Btx A. 32 Patienten hatten im gleichen Verfahren eine Kochsalzlösung erhalten. Die Prozedur war nach zwölf Wochen wiederholt worden.

Vor den Injektionen hatte der von den Patienten berichtete Schmerzgrad auf einer Skala von 0 (keine Schmerzen) bis 10 (maximal vorstellbarer Schmerz) in der Btx-A-Gruppe bei 6,5 Punkten gelegen - ein Wert, der starke Schmerzen bedeutet. Nach 24 Wochen, also zwölf Wochen nach der zweiten Injektionsrunde, lag der Punktwert bei 4,6, was mittelstarke Schmerzen bezeichnet. In der Placebogruppe war ein Ausgangswert von 6,4 Punkten gemessen worden, nach 24 Wochen waren es 5,8 Punkte.

Ein signifikanter Unterschied beim Anteil von Patienten, die eine Schmerzreduktion um mindestens $50 \%$ verspürten, war nicht zu verzeichnen (26\% nach Btx A und 16\% nach Placebo). Statistisch bedeutsam war die Differenz bei $30 \%$ iger Reduktion der Schmerzen (65\% vs. $25 \%)$.
Neuropathiepatienten mit Allodynie und einer wenig beeinträchtigten Temperaturwahrnehmung sprachen im Durchschnitt besser an als Patienten ohne diese Merkmale. Dies korrelierte damit, dass Responder in Hautbiopsien eine höhere Dichte an intraepidermalen Nervenfasern aufwiesen. Offenbar ist bei solchen Patienten der nozizeptive Input besser erhalten.

Die analgetischen Effekte gingen indessen nicht mit Veränderungen in den Gewebekonzentrationen von Substanz P oder CGRP (Calcitonin Gene-Related Peptide) einher, die an der Schmerzübertragung in afferenten Neuronen beteiligt sind. Attal und Kollegen gaben daher ihre ursprüngliche Annahme zur analgetischen Btx-AWirkung auf, wonach Btx A die Freisetzung von Neurotransmittern blockiert und die neurogene Entzündung hemmt, also direkt an den nozizeptiven Fasern angreift. „Wir postulieren, dass die Analgesie durch Btx A hauptsächlich mit zentralen Mechanismen der Schmerzübertragung in Verbindung steht", schreiben sie. Dies gelte zumindest für Patienten mit chronischen Schmerzkrankheiten.

(Robert Bublak)

Attal N et al., Lancet Neurol 2016, (online first) nicht. Seine mögliche Größenordnung liegt bei etwa 5\% (zwei Beobachtungsstudien, sechs systematische Übersichtsarbeiten/Metaanalysen).

\section{8. „Vitamin D reduziert die Krebsinzidenz und -mortalität ${ }^{\prime \prime}$}

Die Inzidenz von Krebs wird durch Vitamin D nicht gesenkt. Der Einfluss einer Vitamin-D-Supplementation auf die Krebssterblichkeit ist nicht eindeutig geklärt. Schätzungen, die eine Reduktion der Mortalität nahelegen, beruhen auf Daten von geringer Qualität (eine randomisiert-kontrollierte Studie, sechs systematische Übersichtsarbeiten/Metaanalysen).

\section{9. „Höhere Vitamin-D-Dosen sind besser"}

Die Gabe von hochdosiertem Vitamin D ( $\geq 300.000 \mathrm{IU}$ ) sollten nicht empfohlen werden. Daten zeigen, dass sie das Sturzund Frakturrisiko erhöhen. (zwei randomisiert-kontrollierte Studien, eine systematische Übersichtsarbeit)

\section{0. „Der Vitamin-D-Spiegel sollte routinemäßig gemessen werden"}

Die Nahrung mit Vitamin D zu ergänzen, ist in der Allgemeinpopulation eine sichere Maßnahme, die keiner Tests bedarf. Tests können aus klinischen Gründen indiziert sein, etwa bei Erkrankungen der Nebenschilddrüsen. Spiegel $\geq 50 \mathrm{nmol} / \mathrm{l}$ zeigen an, dass hinreichend viel Vitamin D vorhanden ist. (3 Metaanalysen)

Gegenwärtig unterstütze die Nachweislage eine Supplementation von Vitamin D zur Prävention von Stürzen und Frakturen und zur Senkung der Mortalität dies vor allem bei über 70-Jährigen, schreiben Allan und Kollegen zusammenfassend. „Andere Effekte sind nicht bewiesen", konstatieren sie und verweisen auf die mangelnde Qualität und Größe der vorliegenden Studien. Ein Screening der Vitamin-D-Spiegel sei nicht nötig, und die Gabe hoher Dosen solle vermieden werden.

Robert Bublak

Allan GM et al., J Gen Intern Med, (online first) 